\title{
O Papel da Lipemia Pós-Prandial na Gênese da Aterosclerose: Particularidades do Diabetes Mellitus
}

\section{revisão}

\author{
LUIS ULISSES SIGNORI \\ Rodrigo Della MÉa Plentz \\ MARIa CLÁUdia IRIGOYEN \\ BEATRIZ D'AGORD SCHAAN
}

Instituto de Cardiologia do Rio Grande do Sul, Fundação Universidade de Cardiologia IC/FUC (LUS, RDMP \& BD'AS) e Departamento de Fisioterapia, Universidade de Cruz Alta UNICRUZ (LUS \& RDMP), Porto Alegre, RS; e Unidade de Hipertensão, InCor, Hospital das

Clínicas de São Paulo, Faculdade de Medicina da Universidade de São Paulo USP (MCl), São Paulo, SP.

Recebido em 10/17/06 Aceito em 20/1 1/06

\author{
RESUMO
}

A aterosclerose é uma doença multifatorial e complexa, que determina eventos clínicos causadores de morbi-mortalidade significativa, representada pela ocorrência de infarto agudo do miocárdio, angina e morte súbita. Está associada a anormalidades lipídicas, ativação plaquetária, trombose, inflamação, disfunção endotelial, estresse oxidativo e alterações metabólicas da matriz, entre outros distúrbios. Todas essas anormalidades são mais comuns e acentuadas no paciente com diabetes, assim como no estado pós-prandial. Dentre os fatores de risco para doença arterial coronariana que ainda não são efetivamente empregados nas estratégias de prevenção da doença em grandes populações destaca-se a hiperlipemia pós-prandial, possível marcador precoce de anormalidades metabólicas e disfunção vascular não observadas em jejum. Recentes resultados mostram que as alterações que ocorrem após uma única sobrecarga lipídica se relacionam negativamente à função endotelial, sendo que as alterações na reatividade vascular estão fortemente associadas à progressão da aterosclerose e aos eventos cardiovasculares. Essas alterações podem revelar um estado de intolerância às gorduras que já são detectadas em indivíduos saudáveis, antes mesmo que anormalidades em jejum sejam percebidas. Esta revisão aborda a fisiopatologia envolvida na lipemia pós-prandial e sua relação com a aterogênese, com ênfase no diabetes mellitus.

(Arq Bras Endocrinol Metab 2007;51/2:222-231)

Descritores: Lipemia pós-prandial; Hipertrigliceridemia; Disfunção endotelial; Estresse oxidativo; Inflamação; Aterosclerose

\section{ABSTRACT}

The Role of Post-Prandial Lipids in Atherogenesis: Particularities of Diabetes Mellitus.

Atherosclerosis is a complex and multifactorial disease, which determines clinical events that cause significant morbi-mortality, represented by acute myocardial infarction, angina and sudden death. It is associated with lipid disturbances, platelet activation, thrombosis, endothelial dysfunction, inflammation, oxidative stress, altered matrix metabolism, among other disturbances. All these abnormalities are usual and more pronounced in diabetic patients, as well as in the post-prandial state. Among the coronary artery disease risk factors that are not usually employed in clinical practice in the whole population, postprandial hyperlipemia plays a major role, being a possible early marker of metabolic abnormalities and vascular dysfunction not yet seen in the fasting state. Recent results showed that post-oral lipid overload changes are negatively associated with endothelial dysfunction, and vascular reactivity abnormalities are strongly related to atherosclerosis progression and cardiovascular events. These abnormalities could disclose a lipid intolerance state that can be detected in apparently healthy subjects even before fasting abnormalities are seen. This review will deal with the pathophysiology changes involved in post-prandial hyperlipemia and its relationship with atherogenesis, with particular emphasis to diabetes mellitus. (Arq Bras Endocrinol Metab 2007;51/2:222-231)

Keywords: Post-prandial lipemia; Hypertrigliceridemia; Endothelial dysfunction; Oxidative stress; Inflammation; Atherosclerosis 
A DOENÇA ARTERIAL CORONARIANA é a principal causa de morte nos Estados Unidos e outros países ocidentais, incluindo o Brasil (1). Os fatores de risco clássicos para seu desenvolvimento são bem conhecidos — obesidade, tabagismo, hipertensão arterial sistêmica, dislipidemia, sedentarismo, diabetes mellitus - e responsáveis por $90 \%$ dos casos de infarto agudo do miocárdio (2). O diabetes mellitus (DM) gera aumento de 3 vezes do risco da mortalidade por infarto agudo do miocárdio (3). Há dúvidas se a aterosclerose no paciente com DM é um processo que cursa diversamente do indivíduo sem esta patologia, ou se o DM simplesmente acelera o mesmo processo. A identificação de características fisiopatológicas específicas do paciente com DM pode levar a uma abordagem focada na prevenção e controle da doença nestes pacientes.

Mais recentemente, outros fatores de risco têm sido estudados, os chamados fatores de risco emergentes, ou não clássicos. Dentre eles podemos citar a elevação de homocisteína, lipoproteína (a), fibrinogênio, aumento de marcadores inflamatórios (4) e a lipemia pós-prandial, este último um possível marcador precoce de anormalidades metabólicas e disfunção vascular não ainda observadas em jejum (5-7). No estado pós-prandial, um maior tempo de permanência da elevação de lipoproteínas ricas em triglicerídeos pode causar disfunção endotelial (8), menor disponibilidade de óxido nítrico e aumento de estresse oxidativo (9), alterações envolvidas na gênese da aterosclerose. A aterosclerose está associada a anormalidades lipídicas, ativação plaquetária, trombose, inflamação, disfunção endotelial, estresse oxidativo e alterações metabólicas da matriz (10), entre outros distúrbios. Todas estas anormalidades são mais comuns e acentuadas no paciente com DM.

A lipemia pós-prandial tem sido sugerida como fator de risco para doença arterial coronariana $(9,11)$, embora estudos de seguimento em longo prazo que evidenciem clinicamente esta afirmação ainda não tenham sido publicados. A idéia de que a aterosclerose poderia estar ligada ao metabolismo pós-prandial dos lipídios não é nova, pois foi introduzida por Zilversmit em 1979 (12). Em indivíduos saudáveis, observou-se que os triglicerídeos pós-prandiais associaram-se de forma independente dos outros fatores de risco à maior espessura da íntima-média da carótida (EIMC), indicador precoce de aterosclerose (13). No DM tipo 2 (DM2), a excursão pós-prandial de triglicerídeos é exagerada e prolongada (8), associando-se ao aumento da EIMC (7). Familiares de indivíduos diabéticos que não têm diagnóstico de anormalidades no meta- bolismo da glicose já apresentam intolerância lipídica pós-prandial, mesmo com níveis normais de triglicerídeos de jejum (5).

Entre os mecanismos responsáveis pela maior ategerogênese decorrente da lipemia pós-prandial encontram-se o aumento das lipoproteínas ricas em triglicerídeos (LpRT), a hiperglicemia e hiperinsulinemia, o estresse oxidativo, o processo inflamatório e a disfunção endotelial, os quais serão abordados no decorrer desta revisão.

\section{AUMENTO DAS LIPOPROTEÍNAS RICAS EM TRIGLICERÍDEOS}

É bem estabelecido que a ingestão de uma refeição rica em gorduras causa aumento de triglicerídeos plasmáticos, enquanto que as concentrações de colesterol não se modificam significativamente (5). A curva lipêmica pós-prandial é avaliada pela magnitude (duração e/ou extensão) que corresponde ao tempo para o retorno aos valores basais dos triglicerídeos e pela amplitude (e/ou pico) que corresponde ao momento em que ocorre o valor máximo encontrado após sobrecarga lipídica.

Em relação à magnitude da resposta aguda à sobrecarga lipídica em indivíduos normolipêmicos em jejum, há aumento nos valores das concentrações das lipoproteínas ricas em triglicerídeos (LpRT). Em relação ao tempo, estes valores apresentam-se em forma de curva ascendente após $2 \mathrm{~h}$ e, aproximadamente, na 4a hora apresenta seu ápice (14) e, com o retorno aos valores basais próximo à 6a hora (15). Entretanto, em indivíduos com dislipidemia, o pico das LpRT é observado entre a 4 a e a 6 a horas e o retorno aos níveis basais demorando mais tempo (após 8 horas) (16). O mesmo fenômeno acontece em pacientes com resistência à insulina (15) e DM2 $(8,9,15)$, podendo durar até $12 \mathrm{~h}(9)$.

Em relação à amplitude (pico máximo da curva), observa-se que os valores encontrados em indivíduos com dislipidemia são maiores que em indivíduos saudáveis. No entanto, quando os valores das LpRT em jejum são iguais entre indivíduos com intolerância à glicose e com DM2, não há diferenças em relação a indivíduos controles, indicando que a trigliceridemia de jejum é fator determinante da resposta pós-prandial a uma sobrecarga lipídica oral (15). Estudo recente comparou dois grupos de mulheres pós-menopausa sem dislipidemia e com HDLc (colesterol contido na lipoproteína de alta densidade) alto: um com e outro sem história familiar de hipertrigliceridemia, observan- 
do-se que aquelas com história familiar de hipertrigliceridemia tinham maior magnitude da resposta pós-prandial à sobrecarga lipídica (17). É importante saber o estado pré ou pós-menopáusico das mulheres avaliadas quanto à lipemia pós-prandial, tendo em vista poder haver diferenças quanto a estas respostas nesses dois subgrupos de pacientes (18).

Karpe e cols., em 1992 (19), estudaram o efeito de uma sobrecarga lipídica oral sobre os níveis de triglicerídeos, ácidos graxos livres (AGL) e atividade da lipase lipoprotéica (LPL) por $12 \mathrm{~h} \mathrm{em} 10$ indivíduos saudáveis e em 34 pacientes pós-infarto do miocárdio. Os triglicerídeos se elevaram, conforme esperado. Nos indivíduos saudáveis, a amplitude máxima (pico) foi na $3 \underline{a}$ hora e os valores retornaram aos níveis basais na $6 \underline{a}$ hora, enquanto que os pacientes pós-infarto apresentaram uma lenta redução dos triglicerídeos (pico: 5a hora, retorno na 9a hora). A LPL apresentou seu pico de atividade para ambos os grupos na 6a hora, retornando aos seus valores basais ao final do protocolo ( 12 h), sendo que sua atividade nos pacientes era maior que em indivíduos saudáveis. Esta alteração do clearance dos lipídios, após sobrecarga lipídica oral, leva a uma exposição aumentada das células endoteliais às LpRT, possivelmente deletéria.

Um aumento da retenção de LDLc (colesterol contido na lipoproteína de baixa densidade) e VLDLc (colesterol contido na lipoproteína de muito baixa densidade) à parede arterial aumenta a permeabilidade endotelial, facilitando a adesão de monócitos ao endotélio. Citocinas inflamatórias (como TNF- $\alpha$ e IL$1 \beta)$ podem regular a expressão da LPL nas células endoteliais via RNAm (20). A hipertrigliceridemia pós-prandial e a liberação simultânea de AGL durante hidrólise de triglicerídeos são mediados pela LPL endotelial, e o funcionamento inadequado desta enzima pode causar dano a essas células. Estas alterações estão associadas a um aumento passageiro nas concentrações de citocinas pró-inflamatórias, neutrófilos, moléculas de adesão, ativação dos monócitos e plaquetas e o aumento na formação dos radicais livres (11). Em estudo recente, observou-se que, após a sobrecarga lipídica, as partículas de LDL-c apresentam-se com um fenótipo mais aterogênico (pequenas e densas) e têm uma maior afinidade a seus receptores em indivíduos com hipertrigliceridemia do que em controles normais (23).

As alterações decorrentes do aumento das LpRT não dependem apenas da sua elevação (quantitativa), mas também das características qualitativas da dieta (gorduras saturadas, poliinsaturados e monoinsaturadas). Os ácidos graxos saturados, com exceção do ácido esteárico, aumentam os níveis séricos de todas as lipoproteínas, principalmente o LDLc, uma vez que reduzem a síntese e a atividade dos receptores de LDLc pela diminuição da expressão do RNAm e da fluidez da membrana. Dessa forma, os ácidos graxos saturados aumentam os níveis de LDLc por meio da redução de sua depuração na circulação. A elevação da fração LDLc sangüíneo irá favorecer o depósito lipídico nas paredes dos vasos, ocasionando o aparecimento das placas ateromatosas (22). A ingestão de gordura saturada é sugerida como a principal causa alimentar de elevação do colesterol plasmático.

A substituição isocalórica dos ácidos graxos saturados por ácidos graxos poliinsaturados reduz o colesterol total e o LDLc plasmáticos. Entretanto, no período pós-prandial as gorduras poliinsaturadas podem ser tão prejudiciais ao endotélio vascular como as gorduras saturadas. As gorduras poliinsaturadas (n6), além de reduzirem o LDLc, reduzem também o HDLc, induzindo maior oxidação lipídica, quando os efeitos praticamente se neutralizam. Já a ingestão de ácidos graxos (n-3) determina um decréscimo nos eventos cardíacos (23). As gorduras monoinsaturadas são apresentadas como tão efetivas na redução do colesterol plasmático quanto as poliinsaturadas, entretanto sem provocar a oxidação lipídica e a diminuição nas concentrações de HDLc (24).

$\mathrm{Na}$ interpretação destes estudos é importante, portanto, observar qual a proporção dos diferentes tipos de gorduras utilizadas na sobrecarga oral e dieta prévia do indivíduo. De fato, o estudo que avaliou a lipemia pós-prandial em indivíduos que usualmente ingerem grandes quantidades de óleo de oliva (residentes no sul da Europa) vs. pequenas quantidades deste óleo (norte da Europa) mostraram menor resposta das LpRT em indivíduos que o ingerem usualmente pouco (23).

Não se pode desconsiderar os aspectos individuais (genéticos) no tipo de resposta pós-prandial. Em recente revisão, Lopez-Miranda e cols. (25) demonstraram que a variabilidade dos fenótipos envolvida no metabolismo das lipoproteínas interage nas respostas lipêmicas pós-prandiais e esta com a doença arterial coronariana. Dentre estas, destacam-se o polimorfismo dos genes da apolipoproteínas AI, E, B, CI, CIII, AIV, AV, lipoproteína lipase, lipase hepática e outras. Exemplo disto é que a hipertrigliceridemia pós-prandial de indivíduos portadores do polimorfismo da ApoA5 (TC + CC) caracteriza-se por uma amplitude (pico) maior, sugerindo um possível fator de risco cardiovascular (26).

Apesar de os exercícios físicos terem benefícios cardiovasculares comprovados, exercícios aeróbicos de 
moderada intensidade (27) e exercícios isométricos (28) não são capazes de prevenir as alterações agudas nas LpRT pós-prandial.

Destas observações pode-se inferir que a exposição prolongada das paredes dos vasos sangüíneos às partículas remanescentes das LpRT tem explicação fisiopatológica para possível contribuição na aterogênese. Alterações na amplitude e/ou na magnitude da elevação de LpRT após ingestão de uma sobrecarga lipídica podem revelar um estado de intolerância às gorduras ligada ao risco de doença arterial coronariana, o qual não seria detectado pela simples medida de lipídios em jejum.

\section{HIPERGLICEMIA E HIPERINSULINEMIA}

Classicamente são conhecidos os efeitos da insulina sobre o metabolismo dos substratos, mas este hormônio também tem efeitos sobre a função nervosa, hemostasia, metabolismo das lipoproteínas e função vascular (29). A insulina também estimula a síntese de lipídios nos vasos sangüíneos, em particular a síntese do colesterol e do LDLc, que se ligam às células do músculo liso arterial e aos monócitos/macrófagos (35). Dentre os mecanismos envolvidos na aterosclerose do paciente com diabetes, destacam-se aqueles determinados pela hiperglicemia: formação de produtos avançados da glicação não-enzimática (AGEs), aumento da atividade da aldose-redutase decorrentes do aumento de sorbitol e redução de mio-inositol, aumento na formação das espécies reativas de oxigênio e nitrogênio (RONS), estados de oxidação e a ativação da proteína quinase $\mathrm{C}$ via acúmulo de diacilglicerol (30).

Estudo realizado por nosso grupo demonstrou que, frente a uma sobrecarga lipídica oral, a glicose plasmática (avaliada em jejum, 4 e $6 \mathrm{~h}$ ) apresenta-se inalterada em indivíduos saudáveis, entretanto nos pacientes com intolerância à glicose e com DM2 ocorria uma redução durante o período pós-prandial. $\mathrm{Na}$ 4a hora os valores destes dois grupos foram diferentes quando comparados ao jejum e ao grupo controle; já na $6 \mathbf{a}$ hora os pacientes com DM2 apresentam glicose plasmática menor do que seu valor basal e 4a hora, mas maior do que a dos controles. No mesmo estudo, a insulinemia aumentou excessivamente nos pacientes com diabetes $\mathrm{e}$ com intolerância à glicose na 4 a hora, especialmente nos primeiros, retornando aos valores basais na 6 a hora. Entretanto, salienta-se que os indivíduos com intolerância à glicose ainda apresentaram níveis elevados de insulina quando a glicose já havia normalizado (4aㅡora) e os diabéticos sempre apresentam uma amplitude (pico) mais elevada que os demais grupos (15). Salienta-se que a PCR se correlacionou positivamente com as variáveis que refletem resistência à insulina (glicemia, HOMA e medida da cintura) (31). Estes dados sugerem que $\mathrm{a}$ intolerância à glicose $\mathrm{e} a$ intolerância às gorduras ocorrem simultaneamente (15).

Existem boas evidências para suspeitar que o prejuízo de vasodilatação endotélio-dependente está associado com a resistência à insulina $\mathrm{e} / \mathrm{ou}$ à obesidade $(8,32)$, pelo menos em parte, por exposição excessiva de células endoteliais aos ácidos graxos. Os ácidos graxos são armazenados sob a forma de triglicerídeos no músculo esquelético quando a insulinemia estiver simultaneamente elevada (33). Assim como as fibras musculares, as células endoteliais também têm a capacidade de armazenar triglicerídeos (34). McCarty e cols. (2003) propõem que, assim como os músculos (esquelético), as células endoteliais (e os adipócitos) também são expostas simultaneamente aos níveis aumentados de AGL e insulinemia durante o período pós-prandial, pois o aumento da insulina promove a síntese de triglicerídeos dentro das células endoteliais via LPL. Os triglicerídeos, previamente depositados nas células endoteliais, atuam como uma resistência (fonte extra de exposição) à deposição de novos ácidos graxos durante o período pós-prandial (35).

Salienta-se que no período pós-prandial a glicose é a principal fonte de energia e seus níveis plasmáticos são regulados pela ação da insulina. A insulina inibe a ação da epinefrina (sobre a lipase sensível a hormônios) na liberação dos AGL (36), levando à deposição de mais ácidos graxos (dos triglicerídeos) nos tecidos para formar as reservas de energia, sendo que este processo também ocorre nas células endoteliais (35).

\section{ESTRESSE OXIDATIVO}

As espécies reativas do oxigênio são produtos intermediários do metabolismo celular oxidativo. As principais espécies que atuam como sinalizadores moleculares vasculares são $\mathrm{O}$ ânion superóxido $\left(\mathrm{O}_{2}{ }^{\bullet}\right), \mathrm{O}$ peróxido de hidrogênio $\left(\mathrm{H}_{2} \mathrm{O}_{2}\right)$, o radical hidroxil $\left({ }^{\bullet} \mathrm{OH}\right)$ e o peroxinitrito $\left(\mathrm{ONOO}^{-}\right)$. Estes são chamados de radicais livres, embora inapropriadamente, pois alguns deles não são de fato radicais livres $\left(\mathrm{H}_{2} \mathrm{O}_{2} \mathrm{e} \mathrm{o}\right.$ $\left.\mathrm{ONOO}^{-}\right)(37,38)$.

$\mathrm{O} \mathrm{O}_{2}{ }^{\bullet-}$ exerce papel central na função vascular, pois é responsável pela geração de outras espécies reativas. Entretanto, $\mathrm{O}_{2} 2^{\circ}$ parece ser um fraco oxidante direto para mediar a regulação dos efeitos oxidativos 
biomoleculares. Já o $\mathrm{H}_{2} \mathrm{O}_{2}$ também está bastante inerte na ausência de metais. Entretanto, o ONOOrepresenta uma duradoura e instável molécula reativa, sendo responsável pela ativação da via da ciclooxigenase (38). A formação do $\mathrm{O}_{2}{ }^{\bullet}$ leva à menor biodisponibilidade do óxido nítrico (NO) por inativação progressiva deste, pois estes reagem e formam o peroxinitrito $\left(\mathrm{ONOO}^{-}\right)$, um potente e duradouro oxidante, ligando as espécies reativas de oxigênio às de nitrogênio, também chamado de espécies reativas de oxigênio e nitrogênio (RONS). As RONS podem provocar a peroxidação dos lipídios, das proteínas e dos ácidos nucléicos e, conseqüentemente, a apoptose celular (37). As principais fontes produtoras do $\mathrm{O}_{2}{ }^{\bullet}$ no sistema vascular são o metabolismo celular inadequado das enzimas NADPH oxidase, xantina oxidase, eNOS (enzima óxido nítrico sintase) e mieloperoxidase (MPO).

Em oposição às RONS existem os mecanismos de defesa enzimáticos (celulares) e não enzimáticos (extracelulares). As principais enzimas que participam no mecanismo de defesa celular são a superóxido dismutase (SOD), catalase e a glutationa peroxidase $(\mathrm{GPx})$. As defesas não enzimáticas são, principalmente, as vitaminas A, C e E. Portanto, o estresse oxidativo é um estado em que o excesso das RONS superpõe-se ao efeito dos sistemas antioxidantes (39). O estresse oxidativo pós-prandial é caracterizado pela susceptibilidade do organismo ao dano oxidativo após o consumo de uma dieta rica em lipídios e/ou carboidratos (40). Estudos publicados por nosso grupo de pesquisa mostram que uma sobrecarga lipídica oral reduz a capacidade antioxidante total (TRAP) em indivíduos jovens saudáveis (41).

Apesar de ensaios clínicos randomizados não terem demonstrado eficácia na prevenção de eventos cardiovasculares com o uso de anti-oxidantes (42), o estresse oxidativo está presente em cada um dos fatores de risco para aterosclerose (37). Sugere-se que o excesso crônico de fluxo das RONS está vinculado com o início e/ou a progressão da aterosclerose, pois ativam o endotélio vascular a um perfil pró-aterogênico (37). As alterações redox e a formação crônica aumentada dos radicais livres podem ser responsáveis por todos os mecanismos envolvidos no risco aumentado de aterosclerose observados no diabetes (39). O aumento na formação dos $\mathrm{O}_{2}$ - está associado a uma resposta fisiológica à lesão, inflamação e reparação tecidual $(37,38)$. Entretanto, nos estudos com sobrecarga lipídica oral anteriormente citados, fica evidente $\mathrm{O}$ aumento na formação do $\mathrm{O}_{2}{ }^{\bullet-}$, onde se observa a diminuição da capacidade antioxidante $(14,41)$ e, de uma forma indireta, a adição de antioxidantes (vitamina $\mathrm{C} \mathrm{e/ou} \mathrm{E)} \mathrm{à} \mathrm{alimentação} \mathrm{reverte} \mathrm{a} \mathrm{disfunção}$ endotelial $(9,43)$.

\section{INFLAMAÇÃO}

Recentemente, a investigação dos mecanismos que determinam a aterosclerose tem sugerido que um processo inflamatório desempenha papel central no seu desenvolvimento, progressão e desfechos. Esse processo inflamatório causa mudanças estruturais e funcionais da parede de vasos sangüíneos, que conduzem à disfunção endotelial e ao desenvolvimento das lesões ateroscleróticas (44).

$\mathrm{O}$ adipócito já não é visto como um tecido passivo de armazenamento de energia. Ao invés disso, tem sido reconhecido por produzir substâncias hormonalmente ativas, as adipocinas (45). São liberadas pelo adipócito: leptina, TNF- $\alpha$, angiotensinogênio, adiponectina, resistina e inibidor do ativador do plasminogênio-1 (PAI-1). Estas citocinas atuam numa complexa rede que interliga os leucócitos, células endoteliais vasculares, mastócitos, fibroblastos e células-tronco hematopoiéticas, resultando no controle da proliferação, diferenciação e/ou ativação dessas células através de mecanismos parácrinos e autócrinos.

Indivíduos saudáveis, após uma refeição com alto teor de gordura, apresentam aumento significativo nas concentrações de citocinas pró-inflamatórias, TNF- $\alpha$ (56\%), IL-6 (75\%) e das moléculas de adesão (ICAM-1: 40\% e VCAM-1: 29\%), quando comparado com uma refeição com alto teor de carboidratos. Essas alterações podem ser prevenidas com o uso de vitamina E, sugerindo que o estresse oxidativo regula o aumento das citocinas e das moléculas de adesão (46). As concentrações elevadas de moléculas de adesão são associadas com o risco aumentado de doenças cardiovasculares (47) e refletem o dano ao endotélio vascular. A suplementação com antioxidantes reduz a susceptibilidade da oxidação do LDLc e inibe a secreção de citocinas pró-inflamatórias, possivelmente pela inibição do NF-K $\beta$. A transcrição do NF-K $\beta$ regula a expressão de uma variedade de genes, incluindo as citocinas, receptores imunológicos e moléculas de adesão celular. A ativação do NF-K $\beta$ está presente nas lesões ateroscleróticas, sendo esta modulada pelo equilíbrio redox celular, onde os estados pró-oxidantes ativam e os antioxidantes inibem (48).

Ceriello e cols. (2004) investigaram o efeito da ingestão de $75 \mathrm{~g}$ gordura, $75 \mathrm{~g}$ de glicose, e da combinação destas antes e após o uso de $40 \mathrm{mg} /$ dia de sin- 
vastatina por 12 semanas. Os dois tipos de sobrecarga, individualmente, resultaram no aumento plasmático de moléculas de adesão (ICAM-1, VCAM-1, selectinaE) e nitrotirosina (medida indireta da formação do $\mathrm{ONOO}^{-}$) em indivíduos saudáveis e pacientes com DM2. Porém, a combinação de ambas, ingestão de gorduras e glicose, produziu uma amplificação dos níveis desses marcadores no estado pós-prandial. O tratamento com sinvastatina reduziu os níveis das moléculas de adesão e nitrotirosina em 3 a 6 dias, embora a diminuição nas concentrações lipídicas ocorresse apenas 3 meses depois (49).

Também indivíduos saudáveis apresentam aumento passageiro nas concentrações de citocinas pró-inflamatórias, neutrófilos, moléculas de adesão, ativação dos monócitos e das plaquetas, e formação dos radicais livres em resposta à ingestão de sobrecarga lipídica $(11,28,46)$. A ativação plaquetária e a formação dos radicais livres foram evidenciadas em um ensaio clínico comparando três refeições (alto teor de gordura vs. alto teor de gordura + antioxidantes vs. alto teor de carboidratos) onde, 4 horas após a sobrecarga lipídica oral, o aumento das LpRT foi seguido do aumento dos fatores de agregação plaquetária, sendo estas alterações revertidas pelos antioxidantes contidos na dieta (43). Nosso grupo recentemente demonstrou que uma sobrecarga lipídica oral promove a elevação do fibrinogênio (marcador inflamatório) no período pós-prandial em indivíduos com intolerância à glicose em relação aos controles saudáveis (31).

Esposito e cols. (50) realizaram um estudo comparando indivíduos saudáveis e com DM2, submetidos a três dietas (rica em gorduras, rica em carboidratos e pobre em fibras, e a combinação de uma dieta rica em carboidratos e rica em fibras). Já no estado de jejum os indivíduos com DM2 apresentam concentrações de IL-18 superiores aos indivíduos saudáveis, sendo que a adiponectina apresenta-se menor nesta situação. Após a ingestão das três dietas, verificou-se que a sobrecarga lipídica induziu a um aumento da ILl8 e diminuição da adiponectina em ambos os grupos. Já a sobrecarga de carboidratos, associada à maior quantidade de fibras, diminuiu a concentração de IL18 em ambos os grupos. Entretanto, a adiponectina apenas apresentou-se reduzida após o consumo da dieta rica em carboidratos e com baixa quantidade de fibras nos DM2.

A persistente exposição do endotélio aos fatores de risco para as doenças cardiovasculares produz inflamação crônica, caracterizada pelo aumento da adesividade dos leucócitos e das plaquetas, bem como a produção de citocinas pró-inflamatórias (TNF- $\alpha$, IL-1 $\beta$ e
IL-6) e quimiocinas. Estas facilitam o recrutamento de monócitos e linfócitos $\mathrm{T}$, os quais provocam as lesões nos vasos. As citocinas TNF- $\alpha$, IL-1 $\beta$ e IL- 6 regulam a expressão da secreção da fosfolipase $A_{2}$, a qual promove a liberação de mediadores lipídicos que incluem os eicosanóides (prostaglandinas, tromboxanos, leucotrineos e lipoxinas), lisofosfolipídios e fator de ativação plaquetária dentro da placa aterosclerótica (51).

\section{DISFUNÇÃO ENDOTELIAL PÓS-PRANDIAL}

O endotélio está diretamente relacionado à gênese da aterosclerose. Devido à sua localização anatômica estratégica, entre o sangue e os tecidos, as células endoteliais têm a capacidade de perceber as alterações das forças hemodinâmicas e a ação de mediadores químicos produzidos no local e/ou circulantes no sangue, bem como responder a estas alterações com fatores biologicamente ativos. O fenótipo das células endoteliais pode ser alterado por vários fatores, sem necessariamente provocar a lesão endotelial, desencadeando uma série de eventos que, ao longo do tempo, podem culminar com as alterações estruturais na parede arterial levando à formação da placa aterosclerótica (11). A disfunção endotelial se caracteriza pelo desequilíbrio na produção e/ou na sensibilidade dos fatores derivados do endotélio, prevalecendo na aterosclerose a ação dos fatores constritores pró-aterogênicos em detrimento dos fatores de relaxamento derivados do endotélio anti-trombóticas e anti-mitogênicas (32).

Os fatores de relaxamento derivados do endotélio são o $\mathrm{NO}$, prostaciclina $\left(\mathrm{PGI}_{2}\right)$ e fator hiperpolarizante derivado do endotélio (EDHF). Estímulos como o shear stress (estresse de cisalhamento) e/ou excitação por agonistas (por exemplo, acetilcolina) determinam liberação de $\mathrm{NO}$ e $\mathrm{PGI}_{2}$ pelo endotélio. Estes mediadores ativam a guanilato ciclase (GMP) e adenilato ciclase (AMP) respectivamente, resultando em uma diminuição dos níveis intracelulares de cálcio $\left(\mathrm{Ca}^{2+}\right)$ nas células da musculatura lisa vascular, promovendo assim o relaxamento dos vasos sangüíneos (38). Neste sentido, existe ainda o ácido epoxyeicosatrienóico (EETs), o qual promove a abertura dos canais de $\mathrm{Ca}^{2+}$-dependentes do $\mathrm{K}^{+}\left(\right.$canais $\mathrm{K}^{+} \mathrm{Ca}$ ) e ativa a $\mathrm{Na}^{+}-\mathrm{K}^{+}-$ATPase, estimulando a hiperpolarização das células da musculatura lisa vascular a partir do endotélio (52). O EDHF é suprimido quando $\mathrm{NO}$ e $\mathrm{PGI}_{2}$ são liberados pelo endotélio (53).

Em oposição funcional aos fatores de relaxamento, existem os fatores constritores derivados do endotélio, representados pela angiotensina II (Ang II), 
endotelina $(\mathrm{ETl})$, serotonina $(5-\mathrm{HT})$ tromboxano $\mathrm{A}_{2}$ $\left(\mathrm{TxA}_{2}\right)$ e as demais isoformas das prostaglandinas $\mathrm{H}_{2}$ $\left(\mathrm{PGH}_{2}\right)$, que possuem um papel fundamental nas respostas inflamatórias agudas e crônicas, devido às suas ações pró-ateroscleróticas e pró-trombóticas (32).

O NO é o principal fator responsável pelo relaxamento: dependendo da sua concentração, ele pode ativar ou inibir a formação dos demais fatores que atuam no endotélio e, conseqüentemente, nas células musculares lisas vasculares. Suas funções anti-ateroscleróticas incluem: inibição da adesão e migração dos leucócitos, impedimento da agregação plaquetária, redução da permeabilidade endotelial às macromoléculas lipoprotéicas, impedimento do acúmulo sub-endotelial de LDLc e a sua oxidação, prevenção da formação das células espumosas (foam cells), impedimento da proliferação e migração das células musculares lisas vasculares e, ainda, contribuição na vasodilatação dos vasos coronarianos durante o aumento de demandas metabólicas (54).

A geração de $\mathrm{O}_{2} \bullet$ diminui a biodisponibilidade do NO livre, enquanto conduz à formação aumentada de $\mathrm{ONOO}^{-}$. $\mathrm{O} \mathrm{O}_{2}{ }^{--}$capta efetivamente o $\mathrm{NO}$, formando o peroxinitrito $\left(\mathrm{ONOO}^{-}\right)$, que é responsável pela ativação da via ciclooxigenase, ligando os prostanóides à via NO pela sinalização redox (55). A ativação da ciclooxigenase $(\mathrm{COX})$ requer concentrações diferentes de $\mathrm{ONOO}^{-}$para ativar as isoformas desta enzima

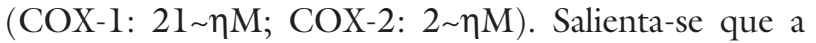
COX-2 é fator crítico na propagação da resposta inflamatória pela vasodilatação, já a COX-1, pela vasoconstrição, favorece o isolamento da área, agregação plaquetária e a coagulação sangüínea. Esse mecanismo das ações das COX sobre a contração muscular é chamado de "tônus das peroxidases", onde primeiramente é ativada a COX-2 e, após uma concentração intracelular 10 vezes maior dos peróxidos, é ativada a COX-1 (56).

O aumento de LpRT e a diminuição do HDLc, característicos da resposta a uma sobrecarga lipídica oral, são fenômenos diretamente relacionados à expressão e à produção da $\mathrm{PGI}_{2}$. Portanto, a ciclooxigenase já está envolvida na disfunção endotelial pósprandial em indivíduos normais. Dados recentes de nosso grupo sugerem que a lipemia pós-prandial interfere no tônus das peroxidases, favorecendo a translocação da COX-2 para COX-1 (57). Resultados semelhantes foram encontrados na avaliação da função endotelial em leito arterial, onde a sobrecarga lipídica oral induziu um aumento da excreção urinária de produtos de modificação oxidativos do ácido araquidônico (14). No diabetes, esse processo cronicamente poderia interferir na deposição endotelial e oxidação do LDLc (58).
Os relatos a respeito das alterações vasculares periféricas, induzidas por sobrecarga lipídica oral, mostraram diminuição na vasodilatação dependente do endotélio e sugerem que a disfunção endotelial transitória esteja ligada à hipertrigliceridemia e/ou à hiperinsulinemia pós-prandial $(9,14,46)$.

Lundman e cols. (59) mostraram que, em homens saudáveis, a infusão venosa de lipídios eleva os AGL $(2 x)$ e triglicerídeos $(4 x)$, e reduz a vasodilatação dependente $(\mathrm{em} 4 \mathrm{x})$ e independente $(2 \mathrm{x})$ do endotélio arterial (dilatação mediada pelo fluxo). Já Anderson e cols. (8) estudaram o efeito da sobrecarga lipídica oral na função endotelial em pacientes com DM2 e controles, mostrando disfunção endotelial em ambos os grupos, com magnitude maior nos pacientes com DM2. Em outro estudo, comparando indivíduos saudáveis com DM2, submetidos a três dietas (rica em gorduras, rica em carboidratos e pobre em fibras, e a combinação de uma dieta rica em carboidratos e rica em fibras), mostrou-se que a refeição de alto teor de gordura ativa o endotélio (avaliado na 4a hora), a coagulação sangüínea e a fibrinólise. Estas alterações foram revertidas pelos antioxidantes contidos na dieta (44).

A elevação pós-prandial de LpRT resulta em disfunção endotelial, provavelmente por mecanismo de aumento de estresse oxidativo, cuja magnitude é maior no diabetes $(8)$, e estão relacionados às complicações crônicas da doença (60). O aumento dos radicais livres está relacionado com o aumento das LpRT, hiperinsulinemia (46) e a redução do HDLc, tornando o LDLc mais aterogênico $(59,63)$, levando à sua deposição e oxidação no espaço sub-endotelial (58), sendo estes mecanismos envolvidos na aterosclerose (8). Baseado nos resultados anteriores, em indivíduos saudáveis, observa-se que a disfunção endotelial que se segue a uma sobrecarga lipídica oral apresenta resultados variáveis de acordo com tipo de refeição e método de avaliação endotelial, sendo que no DM2 essas alterações são mais evidentes.

A seguir, será discutida a interação dos mecanismos possivelmente envolvidos na maior aterogênese, induzidos pelas respostas pós-prandiais à sobrecarga lipídica.

\section{INTERAÇĀO DOS MECANISMOS DE DISFUNÇÃO ENDOTELIAL PÓS-PRANDIAL}

A presente revisão procurou demonstrar que a lipemia pós-prandial está diretamente relacionada aos mecanismos que promovem a aterogênese. As pesquisas relatadas nesta revisão demonstram que os níveis de tri- 
glicerídeos pós-prandiais, e não os provenientes dos adipócitos em jejum, são um marcador mais sensível de aterosclerose.

Os estudos citados mostram que a sobrecarga lipídica induz a um aumento das LpRT, redução do HDLc e à hiperinsulinemia. Esta condição metabólica leva à formação dos radicais livres, que, de acordo com a capacidade antioxidante (endógena e/ou exógena) presente, se reduzida, determina estresse oxidativo. Os radicais livres estimulam os tecidos a secretarem citocinas (TNF- $\alpha$, IL- $1 \beta$ e IL-6), provavelmente através dos macrófagos, estimulando assim a formação das moléculas de adesão $(11,20,51)$. A geração de $\mathrm{O}_{2}{ }^{\bullet}$ diminui a biodisponibilidade do NO livre, resultando na menor vasodilatação dependente do endotélio e também na formação do $\mathrm{ONOO}^{-}$, o qual é responsável pela ativação da via ciclooxigenase (57). Esses processos estão vinculados à gênese e progressão da lesão aterosclerótica (44).

Salienta-se que as alterações na vasodilatação dependentes do endotélio $(9,41)$ que seguem uma sobrecarga lipídica oral em indivíduos saudáveis são um fenômeno tempo-dependente (14) e possivelmente levam à perpetuação destas respostas na aterosclerose, devido à presença dos demais fatores de risco para as doenças cardiovasculares (11).

\section{CONSIDERAÇÕES FINAIS}

O homem moderno vive no estado pós-prandial a maior parte do dia, o que é muito diferente do que ocorria quando vivia de caça-coleta. Além disso, a composição da dieta ingerida passou de rica em fibras e carboidratos para rica em gorduras. Estas mudanças associaram-se a efeitos em longo prazo (obesidade, diabetes, dislipidemia, hipertensão arterial sistêmica e conseqüentemente mais doenças cardiovasculares) e efeitos agudos, os quais podem conferir risco cardiovascular extra. O estado pós-prandial caracteriza-se por excursões glicêmicas, hipertrigliceridemia, propensão à oxidação do LDL-c, aumento de marcadores inflamatórios, hiperatividade simpática e estresse oxidativo, todos fatores potencialmente determinantes de disfunção endotelial, a qual está intimamente ligada à aterogênse.

\section{REFERÊNCIAS}

1. American Heart Association. Heart and stroke statistical update. Dallas: American Heart Association, 2001.

2. Yusuf S, Hawken S, Ounpuu S, Dans T, Avezum A, Lanas F, et al. Effect of potentially modifiable risk factors associated with myocardial infarction in 52 countries (the INTERHEART study): case-control study. Lancet 2004;364:937-52.
3. Stamler J, Vaccaro O, Neaton JD, Wentworth D. Diabetes, other risk factors, and 12-yr cardiovascular mortality for men screened in the Multiple Risk Factor Intervention Trial. Diabetes Care 1993;16:434-44.

4. Ridker PM, Buring JE, Cook NR, Rifai N. C-reactive protein, the metabolic syndrome, and risk of incident cardiovascular events: an 8-year follow-up of 14,719 initially healthy American women. Circulation 2003;107:391-7.

5. Axelsen M, Smith U, Eriksson JW, Taskinen MR, Jansson PA. Postprandial hypertriglyceridemia and insulin resistance in normoglycemic first-degree relatives of patients with type 2 diabetes. Ann Intern Med 1999;131:27-31.

6. Boquist S, Ruotolo G, Tang R, Bjorkegren J, Bond MG, de Faire $U$, et al. Alimentary lipemia, postprandial triglyceriderich lipoproteins, and common carotid intima-media thickness in healthy, middle-aged men. Circulation 1999:100:723-8.

7. Teno S, Uto Y, Nagashima H, Endoh Y, Iwamoto Y, Omori Y, et al. T Association of postprandial hypertriglyceridemia and carotid intima-media thickness in patients with type 2 diabetes. Diabetes Care 2000;23:1401-6.

8. Anderson RA, Evans ML, Ellis GR, Graham J, Morris K, Jackson SK, et al. The relationships between post-prandial lipaemia, endothelial function and oxidative stress in healthy individuals and patients with type 2 diabetes. Atherosclerosis $2001 ; 154: 475-83$.

9. Plotnick GD, Corretti MC, Vogel RS. Effect of antioxidant vitamins on the transient impairment of endothelium-dependent brachial artery vasoactivity following a single high-fat meal. JAMA 1997;278:1682-6.

10. Libby P. Inflammation in atherosclerosis. Nature 2002; 420:868-74.

11. Burdge GC, Calder PC. Horizons in nutritional science plasma cytokine response during the postprandial period: a potential causal process in vascular disease? Br J Nutr 2005;93:3-9.

12. Zilversmit DB. Atherogenesis: a postprandial phenomenon. Circulation 1979;60:473-85.

13. Sharrett AR, Chambless LE, Heiss G, Paton CC, Patsch W. Association of postprandial triglyceride and retinyl palmitate responses with asymptomatic carotid artery atherosclerosis in middle-aged men and women. The Atherosclerosis Risk in Communities (ARIC) Study. Arterioscler Thromb Vasc Biol 1995; 15:2122-9.

14. Tsai W, Li Y, Lin C, Chao T, Chen J. Effects of oxidative stress on endothelial function after a high-fat meal. Clin Sci 2004; 106:315-9.

15. de Ugarte MT, Portal VL, Dias AA, Schaan BD. Metabolic response to oral lipid overload in diabetes and impaired glucose tolerance. Diabetes Res Clin Pract 2005;69:36-43.

16. Maggi FM, Raselli S, Grigore L, Redaelli L, Fantappie S, Catapano AL. Lipotrotein remnants and endothelial dysfunction in the postprandial phase. J Clin Endocrinol Metab 2004;89:2946-50.

17. Kolovou GD, Anagnostopoulou KK, Salpea KD, Pilatis ND, Iraklianou S, Grapsa G, et al. Postprandial lipemia in postmenopausal women with high fasting high-density lipoprotein cholesterol. Am J Med Sci 2006;331:10-6.

18. Masding MG, Stears AJ, Burdge GC, Wootton SA, Sandeman DD. Premenopausal advantages in postprandial lipid metabolism are lost in women with type 2 diabetes. Diabetes Care 2003;26:3243-9.

19. Karpe F, Olivecrona T, Walldius G, Hamsten A. Lipoprotein lipase in plasma after an oral fat load: relation to free fatty acids. J Lipid Res 1992;33:975-84.

20. Hirata K, Ishida T, Matsushita H, Tsao PS, Quertermous T. Regulated expression of endothelial cell-derived lipase. Biochem Biophys Res Commun 2000;272:90-3.

21. Noto D, Rizzo M, Barbagallo CM, Cefalu AB, Verde AL, Fayer $F$, et al. Low-density lipoproteins generated during an oral fat load in mild hypertriglyceridemic and healthy subjects are smaller, denser, and have an increased low-density lipoprotein receptor binding affinity. Metabolism 2006; 55:1308-16. 
22. Mustad VA, Etherton TD, Cooper AD, Mastro AM, Pearson TA, Jonnalagadda SS, et al. Reducing saturated fat intake is associated with increased levels of LDL receptors on mononuclear cells in healthy men and women. J Lipid Res 1997;38:459-68.

23. Wang $C$, Chung M, Lichtenstein A, Balk E, Kupelnick B, DeVine $D$, et al. Effects of omega-3 fatty acids on cardiovascular disease. Evid Rep Technol Assess 2004;94:18.

24. Thomsen C, Rasmussen O, Lousen T, Holst JJ, Fenselau S, Schrezenmeir J, et al. Differential effects of saturated and monounsaturated fatty acids on postprandial lipemia and incretin responses in healthy subjects. Am J Clin Nutr 1999;69:1135-43.

25. Lopez-Miranda J, Perez-Martinez P, Marin C, Moreno JA, Gomez P, Perez-Jimenez F. Postprandial lipoprotein metabolism, genes and risk of cardiovascular disease. Curr Opin Lipidol 2006;17:132-8.

26. Kim JY, Kim OY, Koh SJ, Jang Y, Yun SS, Ordovas JM, et al. Comparison of low-fat meal and high-fat meal on postprandial lipemic response in non-obese men according to the 1131 T >C polymorphism of the apolipoprotein A5 (APOA5) gene (randomized cross-over design). J Am Coll Nutr 2006; $25: 340-7$

27. Pfeiffer M, Wenk C, Colombani PC. The influence of 30 minutes of light to moderate intensity cycling on postprandial lipemia. Eur J Cardiovasc Prev Rehabil 2006;13:363-8.

28. Teixeira M, Kasinski N, Izar MC, Barbosa LA, Novazzi JP, Pinto LA, et al. Effects of acute exercise on postprandial lipemia in sedentary men. Arq Bras Cardiol 2006;87:311.

29. Schaan BD, Rabelo ER, Irigoyen MC. Insulina: efeitos cardiovasculares e aplicações terapêuticas. Arq Bras Endocrinol Metab 2004;48:793-802.

30. Koya D, King GL. Protein kinase $C$ activation and the development of diabetic complications. Diabetes 1998;47:859-66.

31. Schaan BD, Portal VL, de Ugarte MT, Dias AA, Hatem DM. Emerging risk factors and early atherosclerosis indices in subjects with impaired glucose tolerance. Diabetes Metab 2005;31:581-7.

32. Bonetti PO, Lerman LO, Lerman A. Endothelial dysfunction: a marker of atherosclerotic risk. Arterioscler Thromb Vasc Biol 2003;23:168-75.

33. Brechtel K, Dahl DB, Machann J, Bachmann OP, Wenzel I, Maier T, et al. Fast elevation of the intramyocellular lipid content in the presence of circulating free fatty acids and hyperinsulinemia: a dynamic $1 \mathrm{H}-\mathrm{MRS}$ study. Magn Reson Med 2001;45:179-83.

34. Culic O, Decking UK, Schrader J. Metabolic adaptation of endothelial cells to substrate deprivation. Am J Physiol 1999;276:C1061-8.

35. McCarty MF. Does postprandial storage of triglycerides in endothelial cells contribute to the endothelial dysfunction associated with insulin resistance and fatty diets? Med Hypotheses 2003;61:167-72.

36. Penev P, Spiegel K, Marcinkowski T, Van Cauter E. Impact of carbohydrate-rich meals on plasma epinephrine levels: dysregulation with aging. J Clin Endocrinol Metab 2005;90:6198-206.

37. Loscalzo J. Oxidant stress: a key determinant of atherothombosis. Trigliglyceride-rich lipoproteins and atherosclerosis. Review. Biochem Soc 2003;31:1059-61.

38. Bachschmid M, Schildknecht S, Ullrich V. Redox regulation of vascular prostanoid synthesis by the nitric oxide-superoxide system. Biochem Biophys Res Commun 2005:338:536-42.

39. Vinson JA. Oxidative stress in cataracts. Pathophysiology 2006;13:151-62.

40. Bowen PE, Borthakur G. Postprandial lipid oxidation and cardiovascular disease risk. Curr Atheroscler Rep 2004;6:47784.
41. Signori LU, Silva AMV, Plentz RDM, Moreno HJr, Bianchi P, Irigoyen MC, et al. Função endotelial venosa em resposta e estresse oxidativo em resposta à sobrecarga lipídica oral em voluntários normais. Arq Bras Cardiol 2006;87:22234.

42. Heart Protection Study Collaborative Group. MRC/BHF Heart Protection Study of antioxidant vitamin supplementation in 20,536 high-risk individuals: a randomised placebo-controlled trial. Lancet 2002:360(9326):23-33.

43. Esposito K, Nappo F, Giugliano F, Giugliano G, Marfella R, Giugliano D. Effect of dietary antioxidants on postprandial endothelial dysfunction induced by a high-fat meal in healthy subjects. Am J Clin Nutr 2003;77:139-43.

44. Naghavi M, Libby P, Falk E, Casscells W, Litovsky S, Rumberger J, et al. From vulnerable plaque to vulnerable patient: a call for new definitions and risk assessment strategies, parts I and II. Circulation 2003;108:1664-72.

45. Alaniz MHF, Takada J, Vale MICA, Lima FB. O tecido adiposo como centro regulador do metabolismo. Arq Bras Endocrinol Metab 2006;50:216-29.

46. Nappo F, Esposito K, Cioffi M, Giugliano G, Molinari AM, Paolisso G, et al. Postprandial endothelial activation in healthy subjects and in type 2 diabetic patients: role of fat and carbohydrate meals. J Am Coll Cardiol 2002; 39:1145-50.

47. Hwang SJ, Ballantyne CM, Sharrett AR, Smith LC, Davis CE, Gotto AM Jr, et al. Circulating adhesion molecules VCAM1, ICAM-1, and E-selectin in carotid atherosclerosis and incident coronary heart disease cases: the Atherosclerosis Risk In Communities (ARIC) study. Circulation 1997; 16:4219-25

48. Guha M, Bai W, Nadler JL, Natarajan R. Molecular mechanisms of tumor necrosis factor alpha gene expression in monocytic cells via hyperglycemia-induced oxidant stressdependent and -independent pathways. J Biol Chem 2000;275:17728-39.

49. Ceriello A, Quagliaro L, Piconi L, Assaloni R, Da Ros R, Maier $A$, et al. Effect of postprandial hypertriglyceridemia and hyperglycemia on circulating adhesion molecules and oxidative stress generation and the possible role of simvastatin treatment. Diabetes 2004;53:701-10.

50. Esposito K, Nappo F, Giugliano F, Di Palo C, Ciotola M, Barbieri $\mathrm{M}$, et al. Meal modulation of circulating interleukin 18 and adiponectin concentrations in healthy subjects and in patients with type 2 diabetes mellitus. Am J Clin Nutr 2003;78:1135-40.

51. Menschikowski M, Rosner-Schiering A, Eckey R, Mueller E, Koch R, Jaross W. Expression of secretory group IIA phospholipase $A(2)$ in relation to the presence of microbial agents, macrophage infiltrates, and transcripts of proinflammatory cytokines in human aortic tissues. Arterioscler Thromb Vasc Biol 2000;20:751-62.

52. Fleming I. Cytochrome P450 epoxygenases as EDHF synthase(s). Pharmacol Res 2004;49:525-33.

53. Matoba T, Shimokawa H. Hydrogen peroxide is an endothelium derived hyperpolarizing factor in animals and humans. $\mathbf{J}$ Pharmacol Sci 2003;92:1-6.

54. Honing MLH, Morrison PJ, Stroes ESG, Rabelink TJ. Endothelial function in diabetes: a disturbed vascular balance. Internat Diab Rev 1999;8:6-10.

55. Frein D, Schildknecht S, Bachschmid M, Ullrich V. Redox regulation: a new challenge for pharmacology. Biochem Pharmacol 2005;70:811-23.

56. Kulmacz RJ. Regulation of cyclooxygenase catalysis by hydroperoxides. Biochem Biophys Res Commun 2005:338:25-33.

57. Signori LU, Silva AMV, Irigoyen MC, Schaan BD. Papel da ciclooxigenase na reatividade vascular venosa em resposta à sobrecarga lipídica oral (SLO) em voluntários saudáveis. Arq Bras Cardiol 2006;87:221. 
58. Makimattila S, Liu ML, Vakkilainen J, Schlenzka A, Lahdenpera $\mathrm{S}$, Syvanne M, et al. Impaired endothelium-dependent vasodilatation in type 2 diabetes. Relation to LDL size, oxidized LDL, and antioxidants. Diabetes Care 1999;22:973-81.

59. Lundman P, Eriksson M, Schenck-Gustafsson K, Karpe F, Tornvall P. Transient triglyceridemia decreases vascular reactivity in young, healthy men without risk factors for coronary heart disease. Circulation 1997;96:3266-8.

60. Heine RJ, Dekker JM. Beyond postprandial hyperglycaemia: metabolic factors associated with cardiovascular disease. Diabetologia 2002;45:461-75.
Endereço para correspondência:

Beatriz D'Agord Schaan

Unidade de Pesquisa do IC/FUC

Av. Princesa Isabel 370

90620-001 Porto Alegre, RS

Fax: (51) 3230-3600 R.3777

E-mail:editoracao-pc@cardiologia.org.br

/ beatrizschaan@terra.com.br 